\title{
ANOMALY DETECTION OF TRAFFIC MONITORING IN WIRELESS SENSOR NETWORKS(WSN) - A SURVEY
}

\author{
Kanimozhi J. \\ Department of computer science \\ Pondicherry University \\ Pondicherry, India
}

\author{
Dr. M. Nandhini (Assistant Professor) \\ Department of computer science \\ Pondicherry University \\ Pondicherry, India
}

\begin{abstract}
Wireless sensor network (WSN) it faces a heavy traffic within the sensor nodes, which causes various issues. In order to view this issue in wireless sensor network (WSN) we undergo anomaly detection. A traffic monitoring application is chosen to monitor the traffic and to detect anomaly. Anomaly is been detected using various techniques proposed in WSN. A comparative table is made to compare the various techniques.
\end{abstract}

Keywords: wireless sensor network (WSN); traffic monitoring; VANET; outlier.

\section{INTRODUCTION}

\section{A. Wireless sensor networks (WSN):}

Wireless sensor network (WSN) is a network which consists of spatially distributed autonomous sensors [6]. It consists of single node to hundreds of node, these nodes are connected among themselves and they are been integrated with sensing, computational power, and short range wireless communication capability [10]. But WSN are vulnerable to fault and malicious attacks

\section{B. VANET:}

Vehicles connected to each other through an ad-hoc formation in a form of wireless network or wireless sensor network is considered to be as vehicular ad-hoc network (VANET) [11]. Vehicular ad-hoc networks (VANET) are a subgroup of mobile ad-hoc network (MANET). It includes V2V (vehicle to vehicle) communication or V2R (vehicle to roadside) communication and is an important concept of intelligent transportation system (ITS). Nodes in VANET communicate through a standard IEEE 802.11p which is a standard for wireless communication [11].

\section{Traffic monitoring in VANET:}

Road traffic monitoring is considered to be as one of the major issues in intelligent transport system (ITS) in VANET [1]. In VANET there are new technologies which are been raised for traffic monitoring like ATMS (advance traffic monitoring system). This traffic monitoring system helps in monitoring the subsystem where if vehicles have been caught in a traffic stream and, at the same time a communication system which allows vehicle to exchange information with each other regarding vehicle speed and current traffic speed [11].

\section{Anomaly detection in traffic monitoring:}

Outlier is considered to be as certain pattern which deviates from the normal pattern. In case of traffic monitoring, outlier is defined as "certain values which exceeds the predefined threshold values" [6]. In traffic monitoring outliers are detected within the set of road segments and not within the single data. As we see in fig 1.

Outlier can be defined as a pattern which deviates from the normal pattern [11]. The outlier detection in wireless sensor network (WSN) is analyzed out of its sources of outlier, types of outlier and techniques for detecting them. To understand the behavior of outlier, different applications such as environmental monitoring, habitat monitoring, healthcare monitoring, industrial monitoring etc., are studied and analyzed. From the knowledge observed out of this outlier detection on traffic monitoring in VANET is motivated and a detailed survey on this is attempted with various parameters such as the techniques to detect the traffic, sources of outlier, the techniques to detect the outlier and the issues to be faced

The remainder of this paper is organized as follows. Section II outlier detection in wireless sensor networks, Section III outlier detection on traffic monitoring in VANET, Section IV conclusion, Section V references.

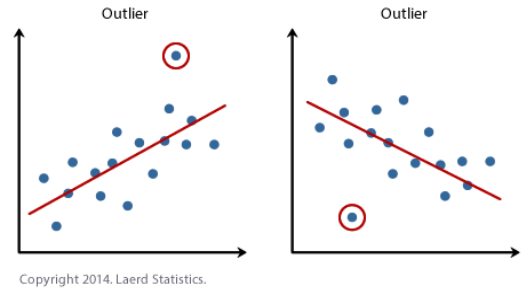

Fig 1. Outlier (the node which deviates from normal node)

\section{OUTLIER DETECTION IN WIRELESS SENSOR NETWORK(WSN)}

\section{A. Wireless sensor network(WSN):}

As said wireless sensor networks are networks which consist of spatially distributed sensors. There may be one or millions of sensors which are connected among themselves to monitor a particular event [6]. In WSN outlier can be defined as "those measurements that significantly deviate from the normal pattern of sensed data" [6]. Outliers can be detected using various technologies. This section discusses about the sources of outlier, the types of 
outlier, and the technique used to detect outlier in wireless sensor networks.

\section{B. Sources of outlier:}

Sources of outliers are of three types they are 1.noise \& error, 2.event and 3. Malicious attacks. Based on the source of outlier various techniques are applied to detect outlier in wireless sensor networks [6] (WSN).

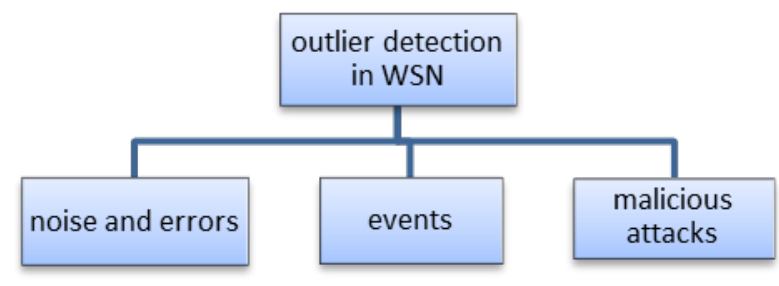

Fig 2. Source of outliers in WSN

\section{1) Noise \& errors:}

Noise \& error refers to a measurements coming from a faulty sensors [10]. They may occur due to misbehaviors of sensors or sensor faults. They have a higher probability of occurrence when compared to events. The probability of noise \& error is maximum within a harsh environment [19]. Data qualities can be affected by these errors so they need to be detected and corrected where it could be used in some other process. E.g. landslide monitoring, coal mining etc.

\section{2) Events:}

A process or phenomena which changes the state of environment is considered to be as event, they are neither noise nor error but they deviate from the normal data set [10]. The event with higher probability is identified using event detection technique. Event measurements are spatially correlated or temporally correlated. E.g., chemical spill, traffic monitoring etc., it changes the historical pattern and lasts for long time [16].

\section{3) Malicious attacks:}

It is an attempt to forcefully abuse someone's device like computers through computer virus, phishing etc., and its motto is stealing the personal information or slow down the function of the system [6]. Malicious attacks consist of some security threats such as communication attack, node compromise, denial of service attack, impersonation attack, and protocol specific attack [10].

\section{Types of outlier:}

Based on the sources of outlier, the types of outlier is been described which helps in analyzing the different techniques used to detect outliers in WSN [20]. Types of outlier are considered to be as very important aspect of outlier detection technique. The types of outlier include 1. Point outliers 2. Contextual outliers 3. Collective outliers

\section{1) Point outliers:}

It is the simplest form of outlier. Point outlier is defined as "Any particular point in a statistical reading which deviates from the normal set of data”. E.g., credit card reading, here outlier is been calculated based on the amount spend, if the amount is considered to be as large then it is considered to be as outlier [20].

\section{2) Contextual outliers:}

Contextual outliers are based on the context (condition) of the data. It mostly depends on the temporal correlations of data [19]. Contextual outliers are calculated mostly on the comparison of neighbors. E.g., a six feet adult is normal, but if it is a six feet child then it is abnormal, hence considered to be as outlier. Contextual outliers are based on two attributes [20].

a) Contextual attributes:

Contextual attributes are completely based on the context, where they are been compared with their neighbor's.

\section{b) Behavioural attributes:}

It is a non-contextual character of an instance. It is just about any particular data and is not been compared with any of its neighbors.

\section{3) Collective outliers:}

If a data collection is completely anomalous from the entire data set then termed as collective outliers. Collective outliers occur only within the set of data not within a single data [6]. They are present both in temporal and spatial correlations. E.g., the electro cardiogram reading of human heart beat [10].

\section{Outlier detection technique in wireless sensor network (WSN).}

This section describes about the technique used to detect outliers in WSN (wireless sensor networks). It is discussed on the feasibility of the environment based on the applications in wireless sensor network [6]. The technique is classified based on source and type of outlier's in Fig.3. as1. Statistical based techniques 2. Nearest-neighbor based technique 3. Clustering based techniques 4. Classification basedtechniques.

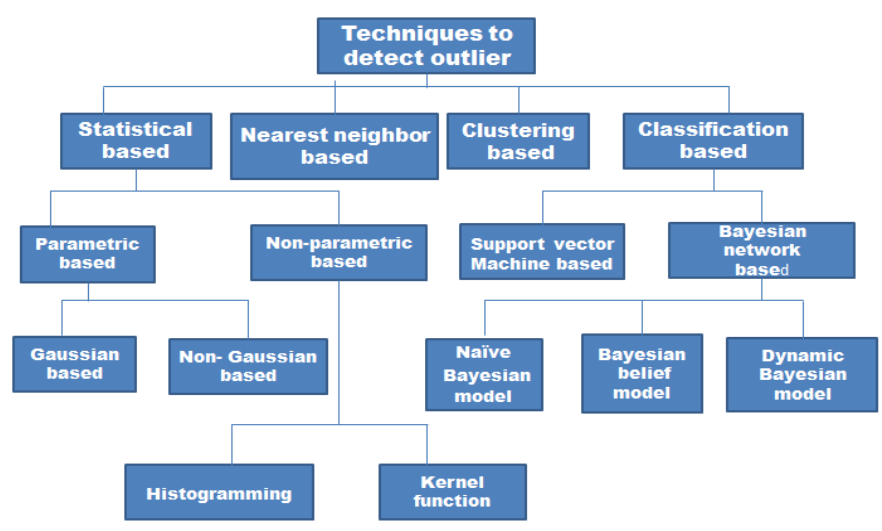

Fig 3. Techniques to detect outlier in WSN

\section{1) Statistical based techniques:}

Statistical based technique is the earliest approach in outlier detection. It is an essential model based technique. It requires a data distribution to detect an outlier [6]. In statistical technique data is been taken on the concept how well they fit the model. It is considered to be as outlier 
if the data instance created by the data set is very low. This technique can work even in an unsupervised mode [19]. The readings in statistical based technique are based on spatial correlation of data. It uses predicted data and threshold distances to detect outlier. Statistical based techniques are classified into 1. Parametric based approaches and 2. Nonparametric based approaches [6]

\section{a) Parametric based approaches:}

In this approach the data obtained is from a known distribution, where the data may be any historical value which is already known [6]. Then the parameter is been classified from the given data. They are further classified into 1. Gaussian-based model and 2. NonGaussian-based model [16]

\section{- Gaussian-based model:}

In Gaussian-based model, the node is detected to be as outlier only if its degree or value of node is always greater than the pre-selected threshold [6]. This technique doesn't work on the temporal correlation of data hence it fails to be relatively high [10].

- $\quad$ Non-gaussian-based model:

In this model a cluster is been detected and they are compared using predicted data and sensing data. Then finally the cluster head collects all the data and detects the outlier [10]. The data which deviates from normal data are considered to be as outlier. This technique is based on spatio temporal correlation. The advantage of this technique is they reduce the computational and communication cost [6].

\section{b) Non-parametric based approaches:}

This technique is not based on availability of data distribution. It is based on distance measure between two instances and threshold value [10]. If the distance between two instances is larger than considered as outlier. It is classified into 1. Histogramming and 2. Kernel functions [11].

\section{- Histogramming:}

This technique uses histogram information for data distribution. Outliers are detected based on fixed threshold distance or rank; [10] it can also be detected by recollecting more histogram information. It reduces the communication cost.

\section{- Kernel functions:}

It uses kernel density estimator for data distribution and it is not based on priori knowledge. Outlier is detected if the values in neighborhood are less than the user specified threshold [6].

\section{2) Nearest neighbor-based approaches:}

It is a widely used approach to analyze data with its neighbor. They use various distance measure to compute the distance between the neighbors. Euclidean distance is a very common approach [6]. Outlier is declared if it is located away from its neighbor. The advantages of nearest neighbor approach include [16]
- The data distribution is not based on the underlying data

- It is a straight forward approach

- It requires only distance measure for calculating data and outlier.

\section{3) Clustering based approaches:}

It is a popular approach. Here clusters are formed based on similar data which have the similar behavior. Outlier is detected if they don't belong to the cluster [6]. Clustering is considered to be as important tool for outlier analysis [10]. Some techniques are based on dense clusters. The advantages of clustering based approach include [16]

- It is easily adaptable, even if the points are added in the middle, they get adapted to detect outliers

- It is highly suitable for detecting temporal data

- It doesn't need a supervision

- Clustering based technique is considered to be the fastest approach.

\section{4) Classification- based approaches:}

It is a systematic approach. There are two phases in classification-based approach the training phase and the testing phase. A set of data is trained using training phase and classified into one of the training classes in testing phase using classification model [19]. During training some data instances lie outside the boundary which is termed to be as outlier. This approach requires no knowledge about available label training. They are further classified into 1.Support vector machine based 2. Bayesian network based [10]

a) Support vector machine based:

This technique separates the data belonging to different class, they are been separated by a hyper plane since they need maximum separation. Outlier can be defined as data which deviates from the hyper plane [10].

\section{b) Bayesian network-based approaches:}

Bayesian network-based approach use a probabilistic graphical model to represent set of variables and their probabilistic independencies. The information which differs from variables are considered to be as outliers. They are further classified into 1 . Naïve Bayesian network model 2. Bayesian belief network model 3. Dynamic Bayesian network models [10]

\section{- $\quad$ Nä̈ve Bayesian network model:}

It is based on spatio-temporal correlation of data. Here each node calculates its incoming node within some subintervals which are divided from the whole value of intervals [19]. The reading which is smaller than other reading in class is considered to be as outlier [6].

\section{- $\quad$ Bayesian belief network model:}

Local outliers are detected using Bayesian belief network model. It is based on spatio temporal correlation [10]. The outlier detected is based on neighborhood reading and its own reading. If the range falls beyond the expected class then it is considered to be as outlier [19].

- Dynamic Bayesian network models: 
It is to identify local outlier in environmental sensor data streams. It is mostly used in dynamic network topology which keeps on changing [19]. This technique is different from other where the outlier is computed if posterior most recent value in sliding window deviates. If the value falls outside the particular interval of values then it is

Literature survey on outlier detection in WSN:

\begin{tabular}{|c|c|c|c|c|c|}
\hline AUTHOR & APPLICATIONS & $\begin{array}{ll}\text { SOURCE } & \text { OF } \\
\text { OUTLIER } & \end{array}$ & TYPE OF OUTLIER & $\begin{array}{l}\text { TECHNIQUE TO } \\
\text { DETECT OUTLIER }\end{array}$ & REMARK \\
\hline $\begin{array}{l}\text { Paulo Gil, et. Al } \\
\text { (2014) [15] }\end{array}$ & $\begin{array}{l}\text { Oil refinery } \\
\text { application }\end{array}$ & $\begin{array}{l}\text { Noise and } \\
\text { errors }\end{array}$ & Point outlier & $\begin{array}{l}\text { Statistical approach } \\
\text { (shewhart control } \\
\text { charts) }\end{array}$ & $\begin{array}{l}\text { Sensitive to } \\
\text { threshold } \\
\text { limits }\end{array}$ \\
\hline $\begin{array}{l}\text { Diego Mendez } \\
\text { et.Al (2012) [6] }\end{array}$ & PS application & $\begin{array}{l}\text { Noise and } \\
\text { errors, } \\
\text { malicious } \\
\text { attack }\end{array}$ & $\begin{array}{l}\text { Point outlier, } \\
\text { Contextual outlier }\end{array}$ & Statistical approach & $\begin{array}{l}\text { To propose new algorithm } \\
\text { to detect large anomalies }\end{array}$ \\
\hline $\begin{array}{l}\text { Osman } \\
\text { et. Al } \\
\text { [12] }\end{array}$ & $\begin{array}{l}\text { Healthcare } \\
\text { monitoring }\end{array}$ & $\begin{array}{l}\text { Noise and } \\
\text { errors }\end{array}$ & Contextual outlier & Statistical approach & $\begin{array}{l}\text { Distributed technology is } \\
\text { used to overcome wastage } \\
\text { of memory }\end{array}$ \\
\hline 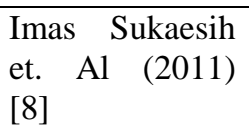 & $\begin{array}{l}\text { Forest fire } \\
\text { indicators }\end{array}$ & Event based & $\begin{array}{l}\text { Contextual outlier, } \\
\text { collective outlier }\end{array}$ & $\begin{array}{l}\text { Clustering } \\
\text { method(based on } \\
\text { neighbor's) }\end{array}$ & $\begin{array}{l}\text { Instead of clustering } \\
\text { method stastical method } \\
\text { can be proposed }\end{array}$ \\
\hline $\begin{array}{l}\text { Juan Ye et. Al } \\
(2015) \text { [18] }\end{array}$ & $\begin{array}{l}\text { Smart home } \\
\text { environment }\end{array}$ & Event based & Point outlier & Statistical approach & $\begin{array}{l}\text { Correlation between } \\
\text { sensors, and between } \\
\text { sensor and activities }\end{array}$ \\
\hline $\begin{array}{l}\text { Umar Ibrahim } \\
\text { Minhas [13] }\end{array}$ & $\begin{array}{l}\text { Underground mine } \\
\text { environment }\end{array}$ & $\begin{array}{l}\text { Noise and } \\
\text { event based }\end{array}$ & collective outlier & Statistical approach & $\begin{array}{l}\text { Vibration pattern analysis } \\
\text { can be added }\end{array}$ \\
\hline $\begin{array}{l}\text { H.D. Kuna a, n } \\
\text { et. Al (2014) } \\
\text { [17] }\end{array}$ & $\begin{array}{l}\text { Outlier detection } \\
\text { in audit logs for } \\
\text { application } \\
\text { systems }\end{array}$ & $\begin{array}{l}\text { Noise and } \\
\text { events }\end{array}$ & Point outlier & $\begin{array}{l}\text { Clustering based } \\
\text { approach }\end{array}$ & $\begin{array}{l}\text { To reduce the level of } \\
\text { false rate even to lower } \\
\text { rate }\end{array}$ \\
\hline
\end{tabular}

\section{E. Application of wireless sensor networks (WSN):}

Outliers are found within various application of wireless sensor network (WSN) [20]. Here we discuss how the outliers are present in some real time applications of (WSN) [6].

\section{1) Environmental monitoring:}

In environmental monitoring sensors are used to sense temperature and humidity of the environment. If outlier is detected it triggers an alarm. Some of the real time application includes traffic monitoring, landslide monitoring [6].

\section{2) Habitat monitoring:}

This sensor used to monitor the behavior of endangered species [20]. Outlier is been detected only if there is some abnormal behavior of the species. Some of its real time application includes monitoring endangered species [9].

\section{3) Healthcare monitoring:}

The sensors are used to monitor the health condition of the body [9]. It helps in detecting the health of the patient. Outliers can be detected if there are some abnormal readings. The application includes sensing body condition of a patient [20].

\section{4) Industrial monitoring:}

They are used in monitoring some machines. Here sensors are used to sense and monitor temperature, pressure, or vibration of the machines. Outlier includes malfunctioning 
with machines. The real time application include oil refinery application

\section{5) Target tracking:}

In target tracking sensors are usually placed on moving target to track their location. Outlier detection is to filter the error information which is used to improve the location of target. This technique increases the accuracy of tracking. The real time application include cloud centric application

\section{OUTLIER DETECTION ON TRAFFIC MONITORING IN VANET}

Traffic monitoring is one of the real time applications in environmental monitoring in wireless sensor networks (WSN). Here traffic monitoring is taken into consideration and we discuss the factors based on traffic like 1. Traffic monitoring 2. Outlier detection of traffic monitoring in VANET. 3. What are the techniques used for detecting outlier in traffic monitoring [21].

\section{F. Traffic monitoring:}

Traffic is considered to be as one of the major issue in VANET, it causes issues like accidents and noise pollution [11]. In VANET traffic arises due to noise, network error, and due to events in roads. As said in traffic monitoring outliers are considered to be as drastic change from the normal value more specifically outlier detection with road segment does not deal with individual moving objects. It is based on temporal correlation and historical value of a data. As we discussed in wireless sensor network there are three sources which causes outliers in real time application similarly in traffic monitoring all the three sources are used to detect anomaly. They are classified as 1. Fault detection 2. Event detection 3. Intrusion detection [21].

\section{1) Fault detection:}

The fault detection defines noise and error. In traffic monitoring fault detection refers to noise pollution, where noise is also considered to be as one of the major cause for outlier [23].

\section{2) Event detection:}

It describes the number of event present. The event which differs from the normal pattern is considered to be as outlier [6]. In traffic monitoring event refers to the number of vehicles present within the environment. Outlier can be defined as the event which differs from the normal pattern and that causes traffic [23].

\section{3) Intrusion detection:}

It refers to the malicious attack, this occurs due to malicious node present within the particular environment [6]. Here the attacker attacks the malicious node present within the particular environment and gains all authorized information such as location of vehicle etc. [23]

\section{G. Techniques used for detecting traffic and outlier in VANET:}

There are many latest technologies immersed to detect traffic, these techniques work under sensor networks. Few WSN technologies are also applied in traffic monitoring to detect outlier. These techniques help in monitoring the traffic and detecting the outlier. Recently Bluetooth technology is immersed to monitor traffic and detect outlier in a shorter distance communication. For longer distance communication between nodes, more than thousands of nodes are placed which get communicated among themselves. The techniques include.

\section{1) IVHS - Intelligent vehicle highway system} It is to increase the capacity of the existing transportation infrastructure. IVHS helps in finding solution for traffic congestion problems [3].

\section{2) ITS - Intelligent transportation system:}

Recently immersed technology with modern embedded system, along with digital connectivity. This combines people and vehicle in a public infrastructure [11]. It is used in monitoring the traffic flow in intersections. The advantage of this system is

1. It reduces the work load of human operators.

2. It warns the driver about dangerous situation.

\section{3) Infrared laser traffic system:}

It was designed to monitor vehicular road traffic. Here laser sources are used which is costly in nature. A road test was made to check the systems capability and count of vehicle during traffic using laser technology. Here instead of sensor nodes laser sensor nodes is been placed to monitor the vehicle. The advantage of this technique is they are weather resistant. Its geometric defines the length parameter of the vehicle [4].

\section{4) Bluetooth communication:}

The Bluetooth communication was invented for repurpose of any applications. It monitors the vehicle, traffic density and flow. Using Bluetooth we can easily track the mobile using mobile cellular service provider [8]. In this technology Bluetooth is been selected because Bluetooth is a telecommunication industry standard which defines how mobile phones, computers and other electronic device gets communicated with short range [22].

\section{5) CCA(Cooperative collision avoidance):}

This technique is based on IEEE standard. It is based on vehicle to vehicle (V2V) wireless communication. It works under medium access control (MAC) protocol. CCA is been used for safety communications in ITS [10].

6) Video- based traffic monitoring:

It is applied for different scenario such as weather and illumination condition. This video based technique is more powerful because the information is 
associated with image sequence [16]. It deals with vehicle tracking, but faces many disadvantages like

1. They have limited capabilities and are costly

2. It becomes tough to monitor the vehicle at night due to cast shadows, vehicle headlight and noise.
This system helps in broadcasting the movement of vehicles periodically. It reduces the number of vehicle crashes. This technology provides vehicle to vehicle (V2V) and vehicle to infrastructure (V2I) communication [16]. It also broadcasts short messages such as vehicle position, speed, heading, acceleration, brake status etc .

7) WAVE-Wireless access in vehicular environment:

Literature survey on traffic monitoring in VANET:

\begin{tabular}{|c|c|c|c|c|c|}
\hline AUTHOR & APPLICATIONS & $\begin{array}{ll}\text { TECHNIQUE } & \text { TO } \\
\text { DETECT TRAFFIC } & \end{array}$ & $\begin{array}{ll}\text { SOURCE } & \text { OF } \\
\text { OUTLIER } & \end{array}$ & $\begin{array}{l}\text { TECHNIQUE TO } \\
\text { DETECT } \\
\text { OUTLIER } \\
\end{array}$ & ISSUES \\
\hline $\begin{array}{l}\text { Juan (Susan) } \\
\text { pan-2017 [11] }\end{array}$ & $\begin{array}{l}\text { DIVERT- A distributed } \\
\text { vehicular traffic re- } \\
\text { routing system for } \\
\text { congestion avoidance }\end{array}$ & $\begin{array}{l}\text { Privacy-aware traffic } \\
\text { rerouting }\end{array}$ & Event & $\begin{array}{l}\text { Statistical } \\
\text { technique, } \\
\text { clustering based } \\
\text { approach }\end{array}$ & $\begin{array}{l}\text { Scalability } \\
\text { network }\end{array}$ \\
\hline $\begin{array}{l}\text { J. Guevara F. } \\
\text { Barrera [22]- } \\
2011\end{array}$ & $\begin{array}{l}\text { Environmental WSN for } \\
\text { road traffic application }\end{array}$ & $\begin{array}{l}\text { Technique of IEEE 1451, } \\
\text { mobile sensors }\end{array}$ & $\begin{array}{l}\text { Noise \& error, } \\
\text { event, malicious } \\
\text { attacks }\end{array}$ & $\begin{array}{l}\text { Statistical } \\
\text { technique }\end{array}$ & $\begin{array}{l}\text { Scalability } \\
\text { network }\end{array}$ \\
\hline $\begin{array}{lr}\text { David } & \text { H. } \\
\text { Roper } & \text { [3]- } \\
2008 & \\
\end{array}$ & $\begin{array}{lr}\text { Advanced } & \text { traffic } \\
\text { management } & \text { in } \\
\text { California } & \\
\end{array}$ & $\begin{array}{l}\text { IVHS - intelligent } \\
\text { vehicle highway system }\end{array}$ & Event & $\begin{array}{l}\text { Statistical } \\
\text { technique }\end{array}$ & Flow of balancing \\
\hline $\begin{array}{l}\text { Shunsuke } \\
\text { kamijo [11]- } \\
2000\end{array}$ & $\begin{array}{l}\text { Traffic monitoring \& } \\
\text { accident detection and } \\
\text { intersections }\end{array}$ & $\begin{array}{l}\text { ITS } \quad-\quad \text { intelligent } \\
\text { transportation system }\end{array}$ & $\begin{array}{l}\text { Noise \& error, } \\
\text { event }\end{array}$ & $\begin{array}{l}\text { Statistical } \\
\text { technique }\end{array}$ & Flow of traffic \\
\hline $\begin{array}{ll}\text { Jie } & \text { zhou } \\
\text { dashan } & {[11]-} \\
2007 & \\
\end{array}$ & $\begin{array}{lr}\text { Moving vehicles for } \\
\text { automatic } & \text { traffic } \\
\text { monitoring } & \end{array}$ & $\begin{array}{l}\text { Video based traffic } \\
\text { monitoring }\end{array}$ & $\begin{array}{l}\text { Noise \& error, } \\
\text { event, malicious } \\
\text { attacks }\end{array}$ & $\begin{array}{l}\text { Statistical } \\
\text { technique, nearest } \\
\text { neighbor based } \\
\text { approach }\end{array}$ & $\begin{array}{l}\text { Robustness } \\
\text { image }\end{array}$ \\
\hline $\begin{array}{lr}\text { Angele } & \text { di } \\
\text { febbraro } & {[16]-} \\
2006 & \\
\end{array}$ & $\begin{array}{l}\text { A new two level model } \\
\text { for multiclass freeway } \\
\text { traffic }\end{array}$ & $\begin{array}{l}\text { DEDS - discrete event } \\
\text { dynamic system }\end{array}$ & Event & $\begin{array}{l}\text { Statistical } \\
\text { technique }\end{array}$ & $\begin{array}{l}\text { High speed and } \\
\text { traffic density }\end{array}$ \\
\hline $\begin{array}{lr}\text { Tari } & \text { M. } \\
\text { Hussain } & {[4]-} \\
2009 & \end{array}$ & $\begin{array}{l}\text { Overhead infrared } \\
\text { sensor for monitoring } \\
\text { vehicular traffic }\end{array}$ & $\begin{array}{lll}\text { Infrared } & \text { laser } & \text { traffic } \\
\text { system } & & \\
\end{array}$ & $\begin{array}{l}\text { Event, } \\
\text { malicious } \\
\text { attacks. }\end{array}$ & $\begin{array}{l}\text { Clustering based } \\
\text { approach }\end{array}$ & $\begin{array}{l}\text { Vehicle speed } \\
\text { measurements }\end{array}$ \\
\hline
\end{tabular}

\section{CONCLUSION}

In this paper we discussed what outlier, is the sources of outlier, the technique used to detect outlier, and the real time applications of outlier. Here we consider traffic monitoring application to detect traffic, and the technology to detect outlier within traffic. A comparative table is made to compare the different technologies with different applications. Hence it helps in detecting outliers in various real time application of wireless sensor network 


\section{REFERENCES}

[1] J. Guevara1 F. Barrero E. Vargas1 J. Becerra S. Torali, "Environmental wireless sensor network for road traffic application”, 2011

[2] June-ho Bang, Young-Jong Cho, Kyungran Kang, “Anomaly detection in LTE signaling”, 2017

[3] David H. Roper and Goro Endo,"In advanced traffic management in California”, February 1991

[4] Tarik M. Hussain, "Overhead Infrared Sensor for Monitoring Vehicular Traffic”, November 1993

[5] Angela Di Febbraro and Antonella Ferrara , "A New Two-Level Model for Multiclass Freeway Traffic”,1996

[6] Yang Zhang, Nirvana Meratnia, and Paul Havinga, "Outlier detection techniques for wireless sensor networks: a survey", 2010

[7] Jie Zhou, Dashan Gao, and David Zhang, "Moving Vehicle Detection for Automatic Traffic Monitoring”, January 2007

[8] Marc Friesen, Rory Jacob, Paul Grestoni, Tyler Mailey, Marcia R. Friesen, and Robert D. McLeod, "Vehicular Traffic Monitoring Using Bluetooth Scanning Over a Wireless Sensor Network",2013

[9] Asmaa Fawzy, Hoda M.O. Mokhtar, Osman Hegazy, “Outlier detection and classification in wireless sensor networks”, (2013) 14 ,

[10] Nauman Shahid, Ijaz Haider Naqvi, Saad Bin Qaisar, "Characteristics and classification of outlier detection technique for wireless sensor networks in harsh environments: a survey", 16 November 2013

[11] Mario De Felice, Andrea Baiocchi, Francesca Cuouma, Gaetano Fusco and Chiara Colombaroni, "Traffic monitoring and incident detection through VANETs”, 2011
[12] Osman Salem, Yaning Liu, Ahmed Mehaua, and Raouf Boutaba, "Online anomaly detection in wireless body area network for reliable healthcare monitoring”, 2014

[13] Juan Ye, Graeme Stevenson, and Simon Dobson, "Fault detection for binary sensors in smart home environment”, 2015

[14] Yong Wang, Zhip Liu Dianhong Wang, Yamin Li, and Jin Yan, "Anomaly detection and visual parceptions for landslide monitoring based on heterogeneous sensor network”, 2017

[15] Paulo Gil, Amancio Santos, Alberto Cardoso, “An oil refinery application”, 2014

[16] Deepika Pahiya, Romika Yadav, "Outlier detection for different application:” March - 2013

[17] H.D. Kuna, R. Garcia-Martinez, "Outlier detection in audit logs for application systems information systems”, 2014

[18] Etienne Pardoa, David Espesa, Philippe Le Parca, "A framework for anomaly diagnosis in smart home based on ontology", 2016

[19] Asmaa Fauzy, Hoda M.O. Mokthar, Osman Hegazy, "Outlier detection and classification in wireless sensor networks", 2013

[20] Nauman Shahid, Ijas Haider Naqui, Saad Bin Qaisser, "Characteristics and classification of outlier detection technique for WSN in harsh environment -a survey”, 16 November 2012

[21] Subir Biswas, "Vehicle to vehicle wireless communication protocols for enhancing highway traffic safety”, 2011.

[22] Mare Fusen, Rory Tacob, Paul Grestoni, Tyler Mailey, Marcia R. Friesen, and Robert D. Mcleod, "Vehicular traffic Smonitoring using Bluetooth scanning over a WSN”, 2014.

[23] Juan (Susan) Pan, Lulian Sandu Pop and Wistian sBorcea, "DIVERT: a distributed vehicle traffic re-routing system for congestion avoidance”, January 2017 